Review Article

\title{
Holobionts and their hologenomes: Evolution with mixed modes of inheritance
}

\author{
Karen Luisa Haag \\ Departamento de Genética, Instituto de Biociências, Universidade Federal do Rio Grande do Sul (UFRGS), \\ Porto Alegre, RS, Brazil.
}

\begin{abstract}
Symbioses are ubiquitous and have played an influential role in the evolution of life on Earth. Genomic studies are now revealing a huge diversity of associations among hosts and their microbiotas, allowing us to characterize their complex ecological and evolutionary dynamics. The different transmission modes and the asynchronous cell proliferation of the numerous symbionts associated with one host generate a genomic conflict ought to be solved. Two disputing views have been used to model and predict the outcome of such conflicts. The traditional view is based on community ecology, and considers that selection at the level of individuals is sufficient to explain longstanding associations among species. A new perspective considers that the host and its associated microbiota constitute a biological entity called holobiont, and that regarding it as a higher-level unit of selection is unavoidable to understand phenotypic evolution. Novel extended phenotypes are often built through symbiotic interactions, allowing the holobiont to explore and survive in distinct environmental conditions, and may evolve in a Lamarckian fashion.
\end{abstract}

Keywords: Genomics, metagenomics, microbiota, symbiosis, holobiont.

Received: March 29, 2017; Accepted: July 26, 2017.

\section{The hologenome concept and its roots}

You are what you eat, what you live on, what lives on you, and what lives in you (Keeling and Palmer, 2008). The idea that organisms do not evolve independently, but rather in conjunction with all their associated symbionts traces back to writings from the end of the $19^{\text {th }}$ century by Russian evolutionary biologists (see, for example, Mereschkowsky, 1905). 'Symbiogenesis', the evolutionary origin of biological innovations by sybmbiosis, reinforces the role of admixture and hybridization in evolution, rather then isolation and dichotomy (Margulis and Fester, 1991; Khakhina, 1992). The application of genomic tools revealed that all living organisms harbor large and diverse assemblages of symbiotic microorganisms, challenging the common view in which species are the major evolutionary units (Doolittle and Zhaxybayeva, 2010). Recent estimates confirm that bacterial cells outnumber, or at the very least, equal the amount host cells in the human body (Sender et al., 2016). Microbes sustain life on our planet exactly because of their myriad associations and biogeochemical processes; although gnotobiotic animal life is possible inside a bubble, such a condition is known to have deleterious effects on the development of both immune and nervous systems (Gilbert and Neufeld, 2014; Mayer et al., 2015). Most mechanisms

Send correspondence to Karen Luisa Haag. Departamento de Genética, Instituto de Biociências, Universidade Federal do Rio Grande do Sul, Caixa Postal 15053, 91501-970 Porto Alegre, RS, Brazil. E-mail: karen.haag@ufrgs.br. by which symbionts influence the metabolism, physiology, immunity, behavior and development of their hosts are yet to be discovered. Similarly, the evolutionary mechanisms underlying the evolution of symbiotic assemblages remain elusive.

Traditionally, evolutionary biologists have viewed changes within individual genomes as the major source of phenotypic variation leading to adaptation through natural selection, and ultimately generating diversity among species. Mathematical models describing the evolution of symbioses have focused on a restricted number of interacting partners evolving by natural selection at the level of individuals (Yamamura, 1996; Genkai-Kato and Yamamura, 1999; Morris et al., 2012). The 'holobiont', referring to the host and its associated microbiota (Mindell, 1992), and its 'hologenome' i.e., nuclear, organelle and microbiome genomes (Zilber-Rosenberg and Rosenberg, 2008), provide a broader modeling framework by capturing the integrative nature of host-microbiota associations, and demand multilevel selection as well as inheritance of acquired characteristics (Liu, 2011; Bordenstein and Theis, 2015). Thus, theoretical models aiming to characterize holobiont evolution should account for the genome conflicts generated by distinct modes of transmission of microbiota members.

\section{Microbiota transmission and holobiont cohesion}

The holobiont is a complex community that maintains its cohesion by vertical transmission - or recurrent horizon- 
tal acquisition - of a stable microbiota on the one hand, and on the other hand is open to the acquisition of novel microbiota members through horizontal transmission, environmental infection, or host switching (Figure 1). Most symbionts, including mutualists, commensals, parasites and pathogens, have mixed modes of transmission (both vertical and horizontal; Ebert, 2013), which combined with their environmental gain, or random loss, leads to a dynamic pattern of holobiont composition across generations. Nonetheless, hosts with greater genetic divergence may still exhibit more distinguishable microbiota, a pattern called "phylosymbiosis" (Brucker and Bordenstein, 2013; Brooks et al., 2016).

How do symbionts and their hosts establish specific, longstanding associations, without vertical transmission? By characterizing the progression of bacterial colonization of Hydra polyps at various time points and modeling the organizational principles of this colonization process, it was found that the colonization rate depended on local environmental or host-derived factors as well as interactions between individual bacteria (Franzenburg et al., 2013). Such findings could be extended to the process of microbial gut colonization in metazoans, which seems to involve many of the same host factors that are usually involved in attacking bacteria (for example, Toll-like receptors and immunoglobulins), but symbiotic bacteria seem to have certain compounds on their surface that turn this recognition into acceptance rather than attack (Chu and Mazmanian, 2013). Therefore, phylosymbiosis is not necessarily due to coevolution, since it does not imply that members of the microbial community are constant, stable, or vertically transmitted from generation to generation (Brooks et al., 2016).

To understand how the microbiota structure evolves, a rather simplified bi-layered model of microbial composition is normally used (Shapira, 2016), reflecting more or less how tight are the symbiotic associations within the holobiont. Whereas the 'core' microbiota is stable in the host population and is normally composed by a limited number of host-specific primary endosymbionts (P-symbionts; Moya et al., 2008) that may evolve mutualistic interactions with their hosts, the 'shell' microbiota, which includes secondary facultative symbionts ( $\mathrm{S}$-symbionts), is a consequence of shared host-symbiont environment, and may not endure (Figure 1). However, the definition of a core microbiota is not straightforward; it should be taken as a statistical reality that is relative to the sampling effort and to the environment and does not always express the degree of host-symbiont intimacy (Ebert and Qi, 2011). Furthermore, symbionts shifted from an S- to a P-symbiotic lifestyle multiple times in their evolutionary history (Nováková et al., 2009), implying that the distinction between core and shell may change in time as well. Interestingly, the distinction between core and shell is not strictly associated with the symbiont mode of transmission.
Classic examples of vertically transmitted obligate mutualists of the core microbiota are bacteriocyte-associated endosymbionts of plant sap-sucking insects (Figure 1a), such as the gamma-proteobacterium Buchnera aphidicola in aphids (reviewed in Baumann, 2005). Because symbionts are harbored inside a specialized morphological structure, the bacteriocyte, within all (or most) of the insects of a taxonomic group, and since the symbiont is essential for the survival of the host, it is assumed that such associations result from a single ancient infection of an insect. Indeed, the Buchnera-aphid association is estimated to be as old as 230 mya (Ochman et al., 1999). Nevertheless, the core microbiota may also include host-specific symbionts that are recurrently acquired from the environment (Figure 1d), such as the luminous bacterial mutualist Vibrio fischeri of the squid Euprymna scolopes (Nyholm and McFall-Ngai, 2004), or transmitted as an assemblage by other members of the host population (Figure 1e), such as the Gramnegative bacteria that belong to the core honey bee microbiota (Powell et al., 2014). The shell microbiota generally includes free-living opportunistic microorganisms that are common in the environment and may become beneficial or pathogenic. For example, different species of Trichoderma are found free in the soil, but may also associate to plant leaves and roots, protecting them against pathogenic fungi, or even parasitize other fungi (Harman et al., 2004). Other opportunistic fungi include commensal organisms such as different species of Candida that colonize distinct body locations in humans, in particular the gastro-intestinal tract, the genital tract, or the skin, where they may become pathogenic (d'Enfert, 2009). Shell symbionts may also be acquired by host switching, and jump between hosts as distantly related as plants to insects, e.g., Candidatus Phytoplasma, a Gram-positive bacterium that has diverse pathogenic effects on different plant species and is mostly harmless on the insect vectors (Hogenhout et al., 2008).

Holobiont dynamics and evolution is based upon the interactions among all symbionts and their host. The environmental acquisition of a new symbiont may have profound effects on the entire community structure. For example, after ingesting a blood meal containing Trypanosoma cruzi epimastigotes, the diversity of the gut microbiota of the triatomine vector increases, mediated by the insect immune responses (Díaz et al., 2016). In bumble bees it was shown that gut symbionts may even impede the establishment of an immigrant, protecting the holobiont against pathogenic trypanosomes (Koch and Schmid-Hempel, 2012), though this protection seems to rely on a balanced microbiota composition. Some honey bee gut symbionts such as the beta-proteobacterium Snodgrassella alvi are known to protect against trypanosomes, but young bees experimentally fed with additional $S$. alvi are counterintuitively more susceptible to the parasite (Schwarz et al., 2016). 


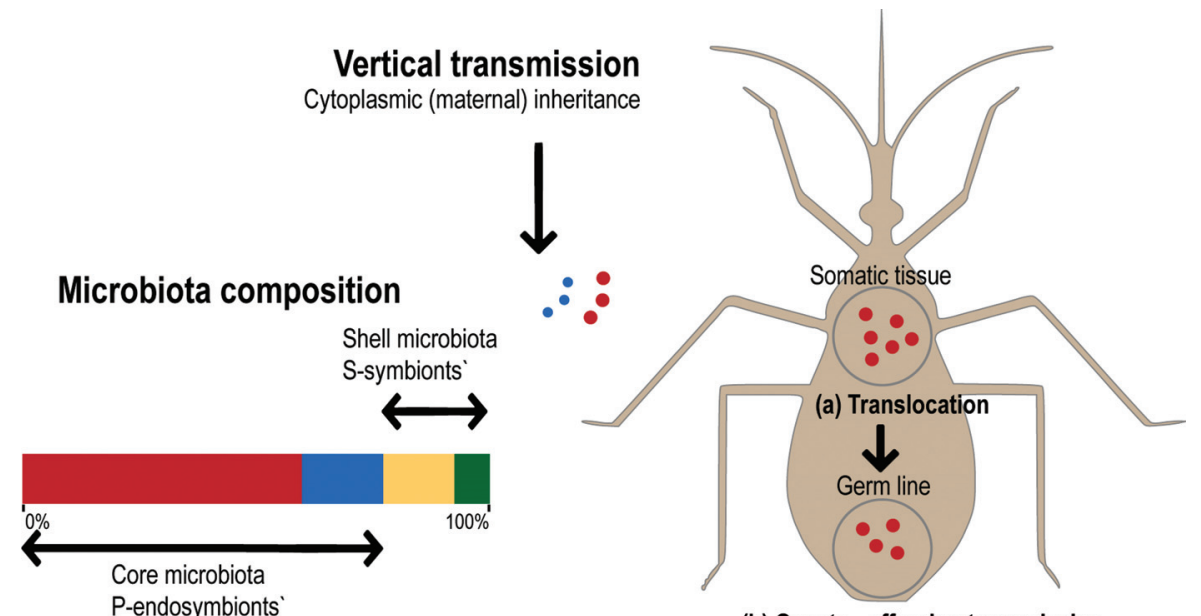

(b) Oocyte - offspring transmission

Horizontal acquisition Inheritance of acquired characteristics

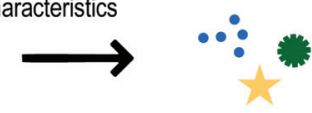

Environment (soil, water, etc.)

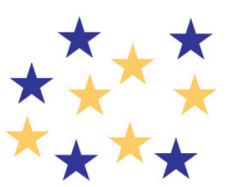

(d) Environmental infection

Host population
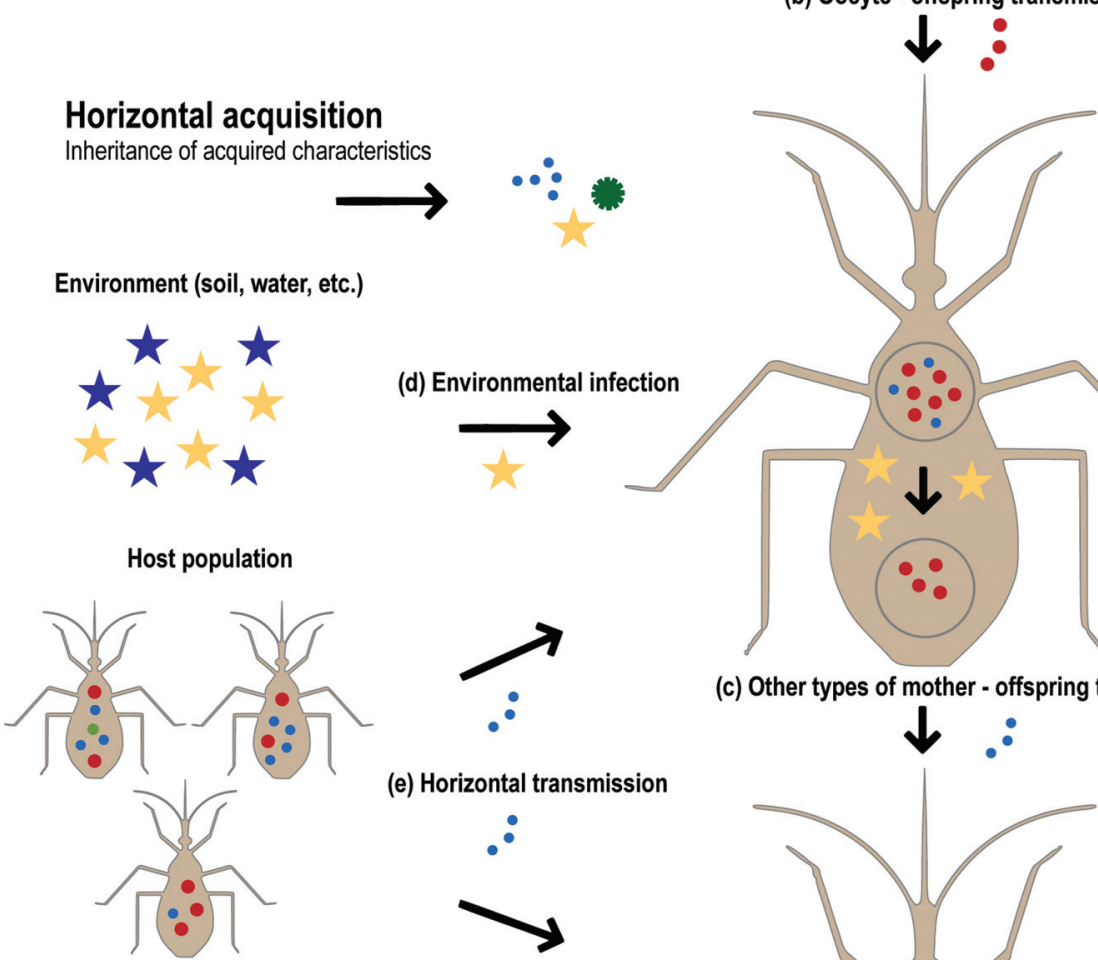

(e) Horizontal transmission

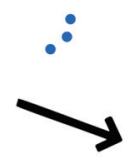

Alternative host
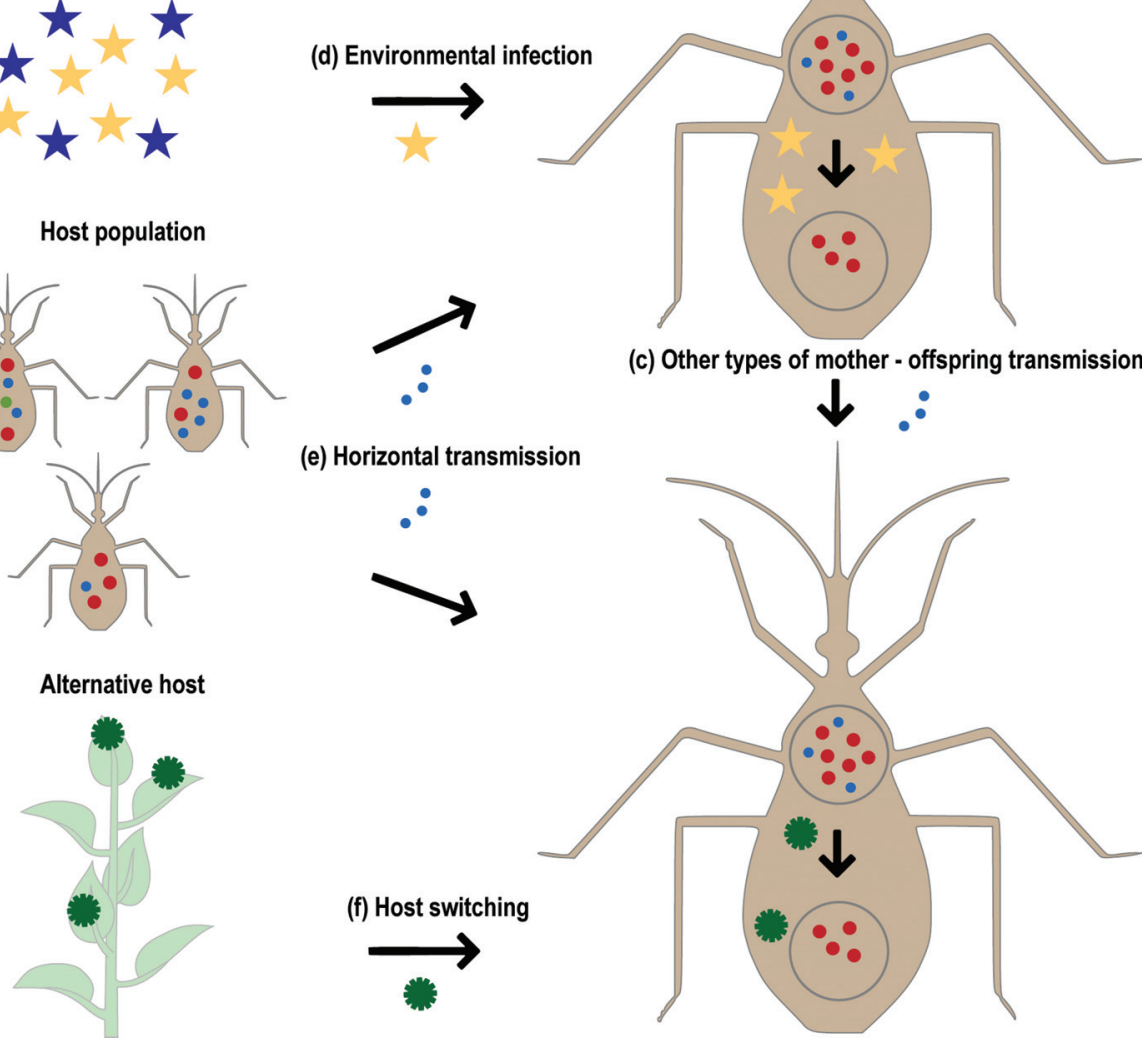

(c) Other types of mother - offspring transmission
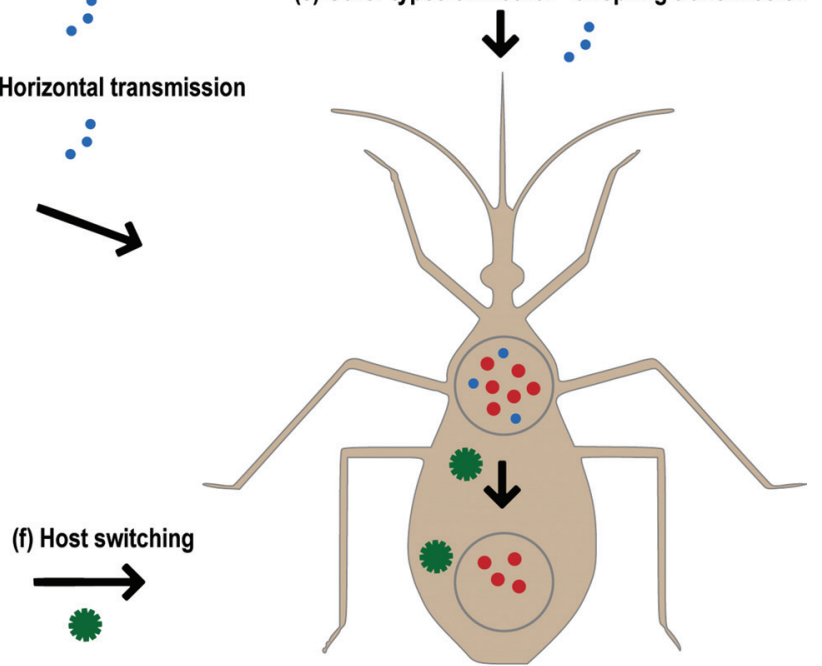

Figure 1 - Modes of microbiota transmission and its bi-layered composition. Vertical transmission is accomplished by the translocation of symbionts from the somatic to the germline tissue (a). Generally the females, which contribute with their oocyte cytoplasm to the zygote, are able to vertically transmit P-endosymbionts, or core microbiota, to their progeny (b). There are numerous other behavioral or physiologic mechanisms by which vertical transmission of P- or S-symbionts might be accomplished (c; reviewed in Funkhouser and Bordenstein, 2013). Both the core and shell microbiota might be horizontally transmitted. Whereas facultative S-symbionts are usually acquired from the environment and might be lost in subsequent generations (d), core symbionts that are acquired from the environment or from other members of the population (e) must be regularly horizontally transmitted or engage some form of vertical transmission to remain in the host population. Symbionts may also switch among distantly related hosts (f) as part of their life cycles or as an opportunistic strategy. Colors of the bar column representing microbiota composition correspond to hypothetical symbionts with distinct modes of transmission as depicted in the figure. 
The complex holobiont dynamics, in addition to the plethora of ways by which it might be disturbed, have lead to numerous criticisms of the holobiont as a unit of selection (Moran and Sloan, 2015; Douglas and Werren, 2016). However, holobiont cohesion might depend less on symbiont transmission modes, or on their shared interests, and more on the specificities of (symbiont $)^{\mathrm{n}} \mathrm{x}$ host interactions and their respective population histories. Associations between host and symbiont genes can be described by the same dynamic model as conventional linkage disequilibria between genes in the same genome, and covariance between host and symbiont genomes depends on geographic structure, selection, and co-transmission rate (Fitzpatrick, 2014).

\section{Genetic inheritance and hologenomic conflict}

'Genomic conflicts', which refer to a dispute of interests caused by the different modes of proliferation and inheritance of distinct genomic segments (Trivers and Burt, 2009) are not confined to symbiotic assemblages. They are found in every replicating eukaryotic cell, i.e., in addition to the classical parent-offspring Mendelian inheritance of nuclear genes that replicate synchronously during mitosis or meiosis, there are various other ways by which genes within one cell are multiplied and transmitted. An individual could be considered to be the product of a successful mutualism of its constituent genes, but sexual reproduction, based on the union of gametes with different genomes, underlies many conflicts, providing conditions for the spread of alleles that help to reduce the competition between different mating types. The fact that most sexual species only have two mating types is intriguing. One hypothesis states that selection at the level of individuals may have favored the spread of nuclear genes that coordinate the inheritance of cytoplasmic genomes - enforcing uniparental inheritance - preventing the competition between unrelated cytoplasmic genomes (Hurst, 1995). Conflicts can even be found within the realm of a single genome. Thus in many species, including humans, more than half of the genome is derived from selfishly replicating transposons (TEs; Koning et al., 2011). The skewed distribution of TEs in most species results from an interplay between evolutionary forces countering TE expansion and host epigenetic transposon silencing mechanisms that evolved by natural selection (Hollister and Gaut 2009). Even though most transposition activity is associated with detrimental phenotypic effects, there are numerous examples of adaptations conferred by TEs that have been domesticated, such as the industrial melanism in Biston betularia (Hof et al., 2016). Furthermore, TEs may have the potential to provide host genomes with the ability to enhance their own evolution (Kidwell and Lisch, 2000).

Besides moving within a single genome, TEs have also the propensity to move across genomes of different species by horizontal gene transfer (HGT; Silva et al., 2004). In eukaryotes, HGT may be accomplished through various routes, ranging from species hybridization (Scavariello et al., 2017) to symbiosis (Schaack et al., 2010). Among prokaryotes HGT seems to be common - estimates based on comparative genomics suggest that up to $15 \%$ of an entire prokaryotic genome might be derived from HGT (Koonin et al., 2001) - and is thought to be a replacement for sex to avoid Muller's ratchet (Koonin, 2011). The utter importance of HGT for bacteria is such, that specialized viral-like particles produced by alpha-proteobacteria called GTAs (Gene Transfer Agents) exist to mediate HGT in coastal and oceanic environments (McDaniel et al., 2010). Not surprisingly, symbionts and their hosts are common HGT players, e.g., parasites may co-opt host genes for their own benefit (Pombert et al., 2015) or vice-versa (Mower et al., 2004). Major transitions in the evolution of life, such as the origin of eukaryotes, had HGT at their roots (Koonin, 2016), and HGT certainly continues to shape how our genomes are and what they do (Koonin et al., 2001; Keeling and Palmer, 2008).

\section{The holobiont as a unit of selection}

Natural selection, increasing the population frequency of beneficial genes, and purging those with detrimental effects, is one of the central principles of current evolutionary theory and explains how genomic conflicts are solved at the level of individuals. Selection at higher levels, such as smaller groups within a population (see for example, Wilson, 1975) or species within a clade (for example, Stanley, 1975), have been viewed with skepticism. The common denominator of all levels of selection is differential survival or reproduction of an inherited feature expressed in a phenotype, no matter where it is manifested, in an individual, population, species, or even a community. Phenotypes are not always reduced to individuals but may constitute a feature that is shared by a group of individuals in the form of 'extended phenotypes' (Dawkins, 1978). Because holobionts essentially do not differ from individuals, neither regarding genomic conflicts, nor with respect to their basic genetic mechanisms of inheritance (Table 1), it is logical to assume that their extended phenotypes are amenable to selection. Analogous to transposable elements and organellar genomes of the host, symbiont genomes within the hologenome can be transmitted horizontally and/or vertically, replicate independently from each other and from the host genome, generating a 'hologenomic conflict'. Moreover, phenotypic novelty might be introduced to the holobiont by a new colonizing symbiont.

Vertically transmitted genes from obligatory mutualists that are harbored in specialized host cells called bacteriocytes need to be translocated into oocytes (Figure 1a). Their transgenerational dynamics is similar to organellar genomes, but their evolution may suffer a stronger effect of genetic drift, due to recurrent bottlenecks caused by translocations (Mira and Moran, 2002). Horizontally inherited symbiont genomes, on the other hand, may be less affected 


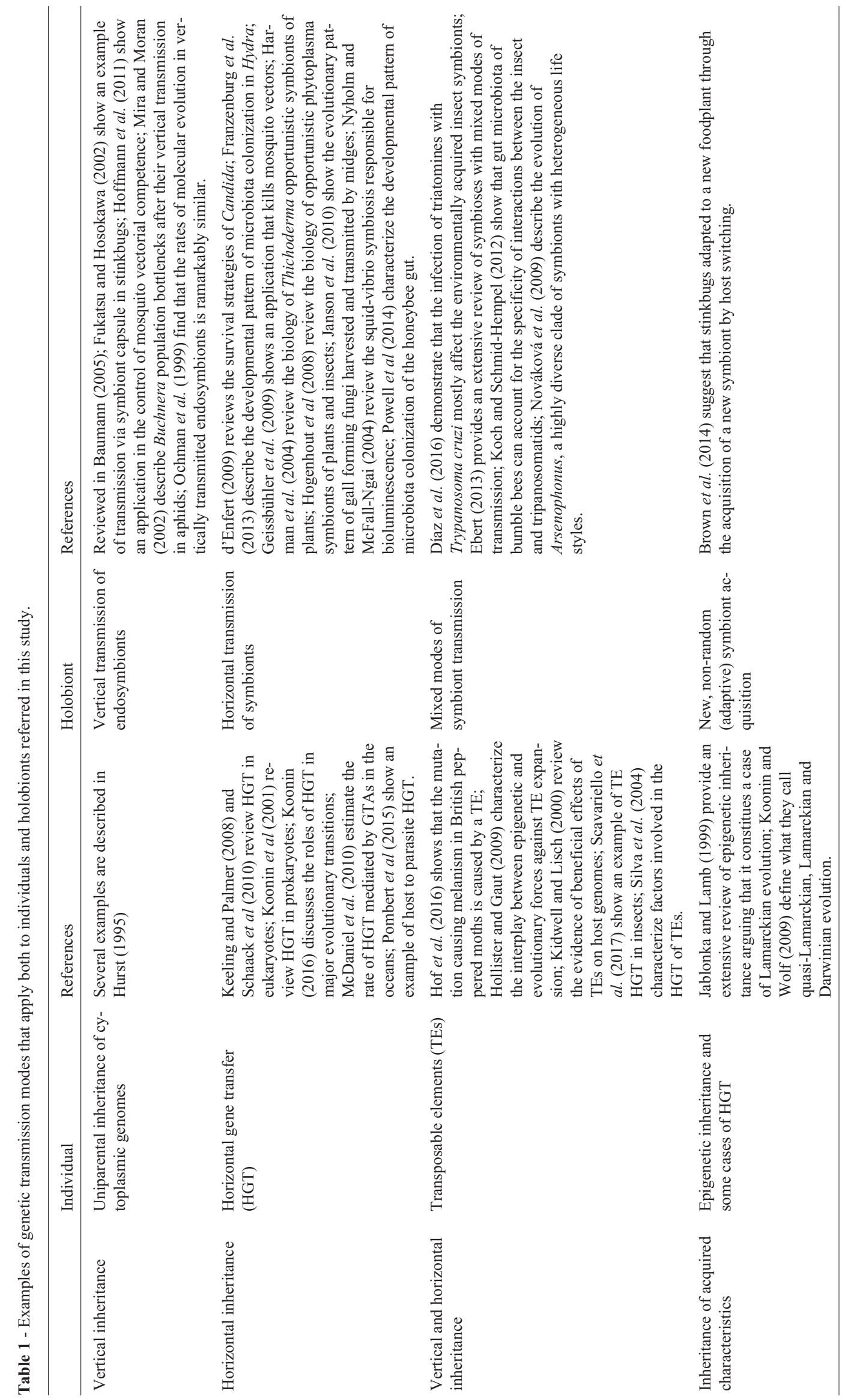


by drift if maintained in large reservoir populations in the environment. It remains to be investigated how the evolutionary factors acting at the level of individuals within symbiont populations interact with the higher-level factors of the holobiont. Intuitively it is expected that genomes of symbionts may proliferate selfishly, compete with other symbiont genomes and end up being eliminated, or being acquired and become incorporated into the hologenome and maintained. Being able model how these selection pressures acting at different holobiont levels interact, based on their directions and intensities, would have numerous applications, particularly in the manipulation of vectorial competence.

Vector-borne diseases are caused by pathogens that must overcome the immune responses elicited by the insect gut microbiota. Symbionts can also directly impair pathogen infectivity or viability, independent of the host, or influence pathogen transmission by altering the host life history (van Tol and Dimopoulos, 2016). Several microbiota-based disease control strategies have been developed for the mosquito vector, including its infection with Wolbachia for dengue control (Hoffmann et al., 2011) and infection with entomopathogenic bacteria such as Bacillus thuringiensis and B. sphaericus that kill mosquito larvae, reducing transmission of malaria (Geissbühler et al., 2009). In paratransgenesis, a mosquito symbiont is genetically manipulated to express a factor that hinders vector competence (Wang and Jacobs-Lorena, 2013). However, to ensure the long-term viability of such strategies, in face of ongoing evolutionary change, the interaction of distinct holobiont selection pressures needs to be dissected.

\section{Inheritance of characters acquired through symbiosis}

The hologenome is a vehicle for the inheritance of acquired characters, because symbiont-induced phenotypes may be adaptive to the host. Let us imagine a gene that is adaptive in a certain environment, e.g., a bacterial gene that allows metabolizing a substance that is common in the environment and is otherwise toxic. The acquisition of such a bacterial symbiont by another organism would allow its survival in such an environment. An advantage induced in an organism by an external (environmental) source, and which is maintained in further generations, is considered 'Lamarckian' evolution, as opposed to the 'Darwinian' form of evolution, which is based on the advantage of random mutations (Jablonka and Lamb, 1999; Koonin and Wolf, 2009). In the context of the hologenome, it is easier to understand adaptations by regarding it as a higher-level unit of selection. I will illustrate this by changes in insect life history traits induced by their symbionts.

Phytophagous stinkbugs from the genus Megacopta maintain nutritional symbionts - the extracellular gammaproteobacterium symbiont 'Candidatus Ishikawaella capsulata' - in the cavity of crypt-bearing posterior midgut, which are vertically transmitted due to a unique translocation mechanism called 'symbiont capsule'. Newly hatched nymphs use their piercing and sucking mouthparts to probe for symbiont-containing capsules laid down with the eggs by their mothers (Fukatsu and Hosokawa 2002). It was shown that the pest status of these insects is principally determined by symbiont genotype rather than by insect genotype. A pest stinkbug species, Megacopta punctatissima, performs well on crop legumes, while a closely related non-pest species, $M$. cribraria, suffers low egg hatch rate on the same plants. When their obligate gut symbiotic bacteria are experimentally exchanged between the species, their performance on the crop legumes is reversed: the pest species shows low egg hatch rate, whereas the non-pest species restores its normal egg hatch rate (Hosokawa et al., 2007). Genomic analyses suggest that the invasion of formerly wild-plant-adapted stinkbugs onto soybean in the US possibly occurred via host switching of the symbiont from unrelated soy-adapted stinkbugs (Brown et al., 2014). Host switching is a likely explanation in this case, because when nymphs are disturbed or capsules are damaged or not found, nymphs rapidly disperse in search of other capsules (Hosokawa et al., 2008). Lamarckian evolution is implicated, because the ability to feed on the crop plant is acquired from the environment, not by random mutations. Furthermore, it is the holobiont, with its hologenome, that evolves via an extended phenotype conferred by the symbiont.

The case above involves a vertically transmitted symbiont that strictly coevolves with the host (Hosokawa et al., 2006). However, neither vertical transmission, nor coevolution is required for hologenome evolution. An interesting example comes from the ectosymbiotic association of the fungus Botryosphaeria dothidea that is harvested by the midge Asteromyia carbonifera inside galls of the host plant Solidago altissima. Striking gall morphologic variation is found sympatrically (in the same habitat) and syntopically (on the same host plant; Crego et al., 1990). Although galls result from the growth of the fungus inside the plant tissue, it is known that gall variation does not derive from the fungus, but rather seems to result from midge 'manipulation'. Genetic studies show that midges from distinct gall morphs are highly differentiated (Stireman et al., 2008), though there is no evidence of gall morph-associated phylogenetic, genetic or phenotypic divergence in the symbiont: there is also no evidence of genomic correlates of a symbiotic lifestyle for the bacterium and essentially no evidence of evolutionary divergence of the symbiotic isolates (midge associated) from free-living (not insect associated) $B$. dothidea populations (Janson et al., 2010). Vertical transmission and reciprocal changes between the fungus and the midge are not implicated in gall morphologic evolution in this association, probably because the fungus is recurrently acquired from the environment and slaved by the midge. Extended 
phenotypes emanating from ecological associations may have strong impacts in community ecology and evolution, suggesting that an integration of ecology and genetics is probably essential for understanding the natural world (Whitham et al., 2003).

\section{Conclusion: why do we need a hologenome?}

The hologenome perspective helps us to pursue the causes of phenotypic variation and evolution. Viewing the holobiont as a unit of selection - not a superorganism - allows understanding that in a complex community of interacting organisms there are emergent (extended) phenotypes. Symbionts are drivers of the evolution of their hosts. They are known to alter the reproduction system and may influence the mating preferences of their hosts, leading to reproductive isolation and speciation (Sharon et al., 2010; Shropshire and Bordenstein, 2016). Symbiont genes may also allow their hosts to explore new environments through extended phenotypes. Invasive species such as sap-feeding insects have to rely on their bacterial symbionts to spread on new host plants (Brown et al., 2014). Similarly, vectorial competence of insects that transmit severe diseases results from the tripartite interaction of host $\mathrm{x}$ microbiota $\mathrm{x}$ pathogen (van Tol and Dimopoulos, 2016), and therefore an emergent property of the holobiont. The reason why the hologenome concept is needed is that it compels us to recognize that phenotypes, which may result from the interaction of many gene products, do not necessarily emerge from a single organism.

\section{Acknowledgments}

Thanks to Dieter Ebert and to two anonymous reviewers for the critical reading of this manuscript. Financial support was received from the Conselho Nacional de Desenvolvimento Científico e Tecnológico (CNPq) and the Fundação de Amparo à Pesquisa do Estado do Rio Grande do Sul (FAPERGS).

\section{References}

Baumann P (2005) Biology of bacteriocyte-associated endosymbionts of plant sap-sucking insects. Annu Rev Microbiol 59:155-189.

Bordenstein SR and Theis KR (2015) Host biology in light of the microbiome: Ten principles of holobionts and hologenomes. PLoS Biol 13:e1002226.

Brooks AW, Kohl KD, Brucker RM, van Opstal EJ and Bordenstein SR (2016) Phylosymbiosis: Relationships and functional effects of microbial communities across host evolutionary history. PLoS Biol 14:e2000225.

Brown AMV, Huynh LY, Bolender CM, Nelson KG and McCutcheon JP (2014) Population genomics of a symbiont in the early stages of a pest invasion. Mol Ecol 23:1516-1530.
Brucker RM and Bordenstein SR (2013) The hologenomic basis of speciation: Gut bacteria cause hybrid lethality in the genus Nasonia. Science 341:667-669.

Chu H and Mazmanian SK (2013) Innate immune recognition of the microbiota promotes host-microbial symbiosis. Nat Immunol 14:668-675.

Crego CL, Weis AE, Polans NO and Bretz CK (1990) Sympatric sibling species from three phenotypically distinct Asteromyia (Diptera: Cecidomyiidae) galls on the same host plant species. Ann Entomol Soc Am 83:149-154.

d'Enfert C (2009) Hidden killers: Persistence of opportunistic fungal pathogens in the human host. Curr Opin Microbiol 12:358-364.

Dawkins R (1978) Replicator selection and the extended phenotype. Z Tierpsychol 47:61-76.

Díaz S, Villavicencio B, Correia N, Costa J and Haag KL (2016) Triatomine bugs, their microbiota and Trypanosoma cruzi: Asymmetric responses of bacteria to an infected blood meal. Parasites Vectors 9:636.

Doolittle WF and Zhaxybayeva O (2010) Metagenomics and the units of biological organization. BioScience 60:102-112.

Douglas AE and Werren JH (2016) Holes in the hologenome: Why host-microbe symbioses are not holobionts. mBio 7:e02099-15.

Ebert D (2013) The epidemiology and evolution of symbionts with mixed-mode transmission. Annu Rev Ecol Evol Syst 44:623-643.

Ebert D and Qi W (2011) An evolutionary ecology perspective on comparative metagenomics. In: Li RW (ed) Metagenomics and Its Applications in Agriculture, Biomedicine, and Environmental Studies. Nova Sciences Publishers, New York, pp 211-236.

Fitzpatrick BM (2014) Symbiote transmission and maintenance of extra-genomic associations. Front Microbiol 5:46.

Franzenburg S, Fraune S, Altrock PM, Künzel S, Baines JF, Traulsen A and Bosch TC (2013) Bacterial colonization of Hydra hatchlings follows a robust temporal pattern. ISME J 7:781-790.

Fukatsu T and Hosokawa T (2002) Capsule-transmitted gut symbiotic bacterium of the Japanese common plataspid stinkbug, Megacopta punctatissima. Appl Environ Microbiol 68:389-396.

Funkhouser LJ and Bordenstein SR (2013) Mom knows best: The universality of maternal microbial transmission. PLoS Biol 11:e1001631.

Geissbühler Y, Kannady K, Chaki PP, Emidi B, Govella NJ, Mayagaya V, Kiama M, Mtasiwa D, Mshinda H, Lindsay SW, et al. (2009) Microbial larvicide application by a largescale, community-based program reduces malaria infection prevalence in urban Dar Es Salaam, Tanzania. PLoS One 4:e5107.

Genkai-Kato M and Yamamura N (1999) Evolution of mutualistic symbiosis without vertical transmission. Theor Pop Biol 55:309-323

Gilbert JA and Neufeld JD (2014) Life in a world without microbes. PLoS Biol 12:e1002020.

Harman GE, Howell CR, Viterbo A, Chet I and Lorito M (2004) Trichoderma species - opportunistic, avirulent plant symbionts. Nat Rev Micro 2:43-56.

Hof AE van't, Campagne P, Rigden DJ, Yung CJ, Lingley J, Quail MA, Hall N, Darby AC and Saccheri IJ (2016) The indus- 
trial melanism mutation in British peppered moths is a transposable element. Nature 534:102-105.

Hoffmann AA, Montgomery BL, Popovici J, Iturbe-Ormaetxe I, Johnson PH, Muzzi F, Greenfield M, Durkan M, Leong YS, Dong Y, et al. (2011) Successful establishment of Wolbachia in Aedes populations to suppress dengue transmission. Nature 476:454-457.

Hogenhout SA, Oshima K, Ammar E-D, Kakizawa S, Kingdom HN and Namba S (2008) Phytoplasmas: Bacteria that manipulate plants and insects. Mol Plant Pathol 9:403-423.

Hollister JD and Gaut BS (2009) Epigenetic silencing of transposable elements: A trade-off between reduced transposition and deleterious effects on neighboring gene expression. Genome Res 19:1419-1428.

Hosokawa T, Kikuchi Y, Nikoh N, Shimada M and Fukatsu T (2006) Strict host-symbiont cospeciation and reductive genome evolution in insect gut bacteria. PLoS Biol 4:e337.

Hosokawa T, Kikuchi Y, Shimada M and Fukatsu T (2007) Obligate symbiont involved in pest status of host insect. Proc $\mathrm{R}$ Soc Lond B-Biol Sci 274:1979-1984.

Hosokawa T, Kikuchi Y, Shimada M and Fukatsu T (2008) Symbiont acquisition alters behaviour of stinkbug nymphs. Biol Lett 4:45-48.

Hurst LD (1995) Selfish genetic elements and their role in evolution: The evolution of sex and some of what that entails. Phil Trans R Soc B-Biol Sci 349:321-332.

Jablonka E and Lamb MJ (1999) Epigenetic Inheritance and Evolution: The Lamarckian Dimension. Oxford University Press, New York, 335 p.

Janson EM, Peeden ER, Stireman JO and Abbot P (2010) Symbiont-mediated phenotypic variation without co-evolution in an insect-fungus association. J Evol Biol 23:2212-2228.

Keeling PJ and Palmer JD (2008) Horizontal gene transfer in eukaryotic evolution. Nat Rev Genet 9:605-618

Khakhina LN (1992) Concepts of Symbiogenesis: A Historical and Critical Study of the Research of Russian Botanists. Yale University Press, New Haven, 177 p.

Kidwell MG and Lisch DR (2000) Transposable elements and host genome evolution. Trends Ecol Evol 15:95-99.

Koch H and Schmid-Hempel P (2012) Gut microbiota instead of host genotype drive the specificity in the interaction of a natural host-parasite system. Ecol Lett 15:1095-1103.

Koning APJ de, Gu W, Castoe TA, Batzer MA and Pollock DD (2011) Repetitive elements may comprise over two-thirds of the human genome. PLoS Genet 7:e1002384.

Koonin EV (2011) The Logic of Chance: The Nature and Origin of Biological Evolution. FT Press, Upper Saddle River, 528 p.

Koonin EV (2016) Viruses and mobile elements as drivers of evolutionary transitions. Phil Trans R Soc B-Biol Sci 371:20150442.

Koonin EV and Wolf YI (2009) Is evolution Darwinian or/and Lamarckian? Biol Direct 4:42.

Koonin EV, Makarova KS and Aravind L (2001) Horizontal gene transfer in prokaryotes: Quantification and classification. Annu Rev Microbiol 55:709-742.

Liu Y-S (2011) Inheritance of acquired characters in animals: A historical overview, further evidence and mechanistic explanations. Ital J Zool 78:410-417.
Margulis L and Fester R (1991) Symbiosis as a Source of Evolutionary Innovation: Speciation and Morphogenesis. MIT Press, Palatino, $459 \mathrm{p}$.

Mayer EA, Tillisch K and Gupta A (2015) Gut/brain axis and the microbiota. J Clin Invest 125:926-938.

McDaniel LD, Young E, Delaney J, Ruhnau F, Ritchie KB and Paul JH (2010) High frequency of horizontal gene transfer in the oceans. Science 330:50-50.

Mereschkowsky C (1905) Über Natur und Ursprung der Chromatophoren im Pflanzenreiche. Biol Centralbl 25:593-604.

Mindell DP (1992) Phylogenetic consequences of symbioses: Eukarya and Eubacteria are not monophyletic taxa. Biosystems 27:53-62.

Mira A and Moran NA (2002) Estimating population size and transmission bottlenecks in maternally transmitted endosymbiotic bacteria. Microb Ecol 44:137-143.

Moran NA and Sloan DB (2015) The hologenome concept: helpful or hollow? PLoS Biol 13:e1002311.

Morris JJ, Lenski RE and Zinser ER (2012) The Black Queen hypothesis: Evolution of dependencies through adaptive gene loss. mBio 3:e0036-12.

Mower JP, Stefanovic S, Young GJ and Palmer JD (2004) Plant genetics: Gene transfer from parasitic to host plants. Nature 432:165-166.

Moya A, Peretó J, Gil R and Latorre A (2008) Learning how to live together: Genomic insights into prokaryote-animal symbioses. Nat Rev Genet 9:218-229.

Nováková E, Hypsa V and Moran NA (2009) Arsenophonus, an emerging clade of intracellular symbionts with a broad host distribution. BMC Microbiology 9:143

Nyholm SV and McFall-Ngai M (2004) The winnowing: establishing the squid-vibrio symbiosis. Nat Rev Micro 2:632-642.

Ochman H, Elwyn S and Moran NA (1999) Calibrating bacterial evolution. Proc Natl Acad Sci U S A 96:12638-12643.

Pombert J-F, Haag KL, Beidas S, Ebert D and Keeling PJ (2015) The Ordospora colligata genome: Evolution of extreme reduction in Microsporidia and host-to-parasite horizontal gene transfer. mBio 6:e02400-14.

Powell JE, Martinson VG, Urban-Mead K and Moran NA (2014) Routes of acquisition of the gut microbiota of the honey bee Apis mellifera. Appl Environ Microbiol 80:7378-7387.

Scavariello C, Luchetti A, Martoni F, Bonandin L and Mantovani B (2017) Hybridogenesis and a potential case of R2 nonLTR retrotransposon horizontal transmission in Bacillus stick insects (Insecta Phasmida). Sci Rep 7:41946.

Schaack S, Gilbert C and Feschotte C (2010) Promiscuous DNA: Horizontal transfer of transposable elements and why it matters for eukaryotic evolution. Trends Ecol Evol 25:537-546.

Schwarz RS, Moran NA and Evans JD (2016) Early gut colonizers shape parasite susceptibility and microbiota composition in honey bee workers. Proc Natl Acad Sci U S A 113:9345-9350

Sender R, Fuchs S and Milo R (2016) Revised estimates for the number of human and bacteria cells in the body. bioRxiv 036103 .

Shapira M (2016) Gut microbiotas and host evolution: Scaling up symbiosis. Trends Ecol Evol 31:539-549.

Sharon G, Segal D, Ringo JM, Hefetz A, Zilber-Rosenberg I and Rosenberg E (2010) Commensal bacteria play a role in mat- 
ing preference of Drosophila melanogaster. Proc Natl Acad Sci U S A 107:20051-20056.

Shropshire JD and Bordenstein SR (2016) Speciation by symbiosis: The microbiome and behavior. mBio 7:e1785-15.

Silva JC, Loreto EL and Clark JB (2004) Factors that affect the horizontal transfer of transposable elements. Curr Issues Mol Biol 6:57-71.

Stanley SM (1975) A theory of evolution above the species level. Proc Natl Acad Sci U S A 72:646-650.

Stireman JO, Janson EM, Carr TG, Devlin H and Abbot P (2008) Evolutionary radiation of Asteromyia carbonifera (Diptera: Cecidomyiidae) gall morphotypes on the goldenrod Solidago altissima (Asteraceae). Biol J Linn Soc 95:840-858.

Trivers R and Burt A (2009) Genes in Conflict: The Biology of Selfish Genetic Elements. Harvard University Press, Cambridge, $601 \mathrm{p}$.

van Tol S and Dimopoulos G (2016) Influences of the mosquito microbiota on vector competence. Adv Insect Physiol 51:243-291.
Wang S and Jacobs-Lorena M (2013) Genetic approaches to interfere with malaria transmission by vector mosquitoes. Trends Biotechnol 31:185-193.

Whitham TG, Young WP, Martinsen GD, Gehring CA, Schweitzer JA, Shuster SM, Wimp GM, Fischer DG, Bailey JK, Lindroth RL, et al. (2003) Community and ecosystem genetics: A consequence of the extended phenotype. Ecology 84:559-573.

Wilson DS (1975) A theory of group selection. Proc Natl Acad Sci U S A 72:143-146.

Yamamura N (1996) Evolution of mutualistic symbiosis: A differential equation model. Res Pop Ecol 38:211-218.

Zilber-Rosenberg I and Rosenberg E (2008) Role of microorganisms in the evolution of animals and plants: The hologenome theory of evolution. FEMS Microbiol Rev 32:723-735.

Associate Editor: Loreta B. Freitas

License information: This is an open-access article distributed under the terms of the Creative Commons Attribution License (type CC-BY), which permits unrestricted use, distribution and reproduction in any medium, provided the original article is properly cited. 